\title{
On the Dirichlet characters of polynomials in several variables
}

\author{
by \\ Wenpeng Zhang and Zhefeng Xu (Xi'an)
}

1. Introduction. Let $q \geq 3$ be an integer and $\chi$ a Dirichlet character modulo $q$. It is a very important problem in analytic number theory to obtain a sharp upper bound for the character sum

$$
\sum_{x=N+1}^{N+H} \chi(f(x))
$$

where $f(x)$ is a polynomial. If $q=p$ is an odd prime, it is a well known consequence of the theorem, due to Weil (see [2]), stating that the Riemann Hypothesis is true for the zeta-function of an algebraic function field over a finite field, that if $\chi$ is a $q$ th-order character to a prime modulus $p$, and if $f(x)$ is not a perfect $q$ th power $(\bmod p)$, then

$$
\sum_{x=N+1}^{N+H} \chi(f(x)) \ll p^{1 / 2} \log p,
$$

where $A \ll B$ denotes $|A|<k B$ for some constant $k$ which in this case depends on the degree of $f$. The estimate (1) is the best possible. In fact, the first author [8] proved the following:

Let $q \geq 3$ be an integer, $\chi$ a primitive character modulo $q$, and $m$ and $n$ two positive integers such that $\chi^{m}, \chi^{n}$ and $\chi^{m+n}$ are also primitive characters modulo $q$. Then for any integers $r$ and $s$ with $(r-s, q)=1$, we have the identity

$$
\left|\sum_{a=1}^{q} \chi\left((a-r)^{m}(a-s)^{n}\right)\right|=\sqrt{q} .
$$

2000 Mathematics Subject Classification: 11L10, 11L20.

Key words and phrases: primitive Dirichlet characters, polynomials, Gauss sums, identity.

This work is supported by N.S.F. (60472068) and P.N.S.F. (2004A09) of P.R. China. 
It is clear that the character sums in (2) can be viewed as Jacobi sums with Dirichlet characters. So the identity (2) provided a new evaluation for the absolute value of some Jacobi sums. In [7], the first author obtained an interesting evaluation formula for this Jacobi sum, by proving the following identity:

$$
\begin{aligned}
\sum_{a=1}^{q} \chi\left((a-r)^{m}(a-s)^{n}\right) & \\
& =\sqrt{q} \chi\left((s m-r m)^{m}(r n-s n)^{n}\right) \bar{\chi}\left((m+n)^{m+n}\right) .
\end{aligned}
$$

For the character sums of polynomials in several variables, Katz [3] studied the similar problem in finite fields, and obtained some sharper estimates for it.

In this paper, as a complement to [7], we will use the properties of Dirichlet character and Gauss sums to study the Dirichlet character sums of polynomials in several variables, and give some more general identities for it. We shall prove the following conclusions:

THEOREM 1. Let $q \geq 3$ be a positive integer with the prime power decomposition

$$
q=\prod_{\substack{p^{\alpha} \| q \\ 2 \nmid \alpha, \alpha>1}} p^{\alpha},
$$

$\chi$ a primitive character modulo $q, k \geq 2, n_{1}, \ldots, n_{k}$ positive odd numbers such that $\chi^{n_{1}}, \ldots, \chi^{n_{k}}$ and $\chi^{n_{1}+\cdots+n_{k}}$ are all primitive characters modulo $q$, and $u=n_{1}+\cdots+n_{k}$. If $\left(n_{1} \cdots n_{k} u, q\right)=1$, then we have the identity

$$
\begin{aligned}
\sum_{a_{1}=1}^{q} & \cdots \sum_{a_{k-1}=1}^{q} \chi\left(a_{1}^{n_{1}} \cdots a_{k-1}^{n_{k-1}}\left(1-a_{1}-\cdots-a_{k-1}\right)^{n_{k}}\right) \\
& = \begin{cases}q^{(k-1) / 2} \bar{\chi}\left(u^{u}\right) \chi\left(n_{1}^{n_{1}} \cdots n_{k}^{n_{k}}\right) \prod_{p \mid q} \varepsilon_{p}^{k-1}\left(\frac{u n_{1} \cdots n_{k}}{p}\right) & \text { if } 2 \nmid q, \\
q^{(k-1) / 2} \bar{\chi}\left(u^{u}\right) \chi\left(n_{1}^{n_{1}} \cdots n_{k}^{n_{k}}\right)\left(\frac{2}{n_{1} \cdots n_{k} u}\right) \prod_{\substack{p \mid q \\
p \neq 2}} \varepsilon_{p}^{k-1}\left(\frac{u n_{1} \cdots n_{k}}{p}\right)\end{cases}
\end{aligned}
$$

where $\varepsilon_{p}=1$ if $p \equiv 1(\bmod 4), \varepsilon_{p}=i$ if $p \equiv 3(\bmod 4)$, and $\left(\frac{m}{n}\right)$ is the Jacobi symbol.

TheOREM 2. Let $q \geq 3$ be a perfect square, $\chi$ a primitive character modulo $q, k \geq 2, n_{1}, \ldots, n_{k}$ positive integers such that $\chi^{n_{1}}, \ldots, \chi^{n_{k}}$ and $\chi^{n_{1}+\cdots+n_{k}}$ are all primitive characters modulo $q$, and $u=n_{1}+\cdots+n_{k}$. If 
$\left(n_{1} \cdots n_{k} u, q\right)=1$, then we have the identity

$$
\begin{aligned}
\sum_{a_{1}=1}^{q} \cdots \sum_{a_{k-1}=1}^{q} \chi\left(a _ { 1 } ^ { n _ { 1 } } \cdots a _ { k - 1 } ^ { n _ { k - 1 } } \left(1-a_{1}-\right.\right. & \left.\left.\cdots-a_{k-1}\right)^{n_{k}}\right) \\
= & q^{(k-1) / 2} \bar{\chi}\left(u^{u}\right) \chi\left(n_{1}^{n_{1}} \cdots n_{k}^{n_{k}}\right) .
\end{aligned}
$$

In particular, for $m=n_{1}=\cdots=n_{k}$, from our theorems we immediately deduce two nice formulas for calculating generalized Jacobi sums:

Corollary 1. Let $q \geq 3$ with the prime power decomposition

$$
q=\prod_{\substack{p^{\alpha} \| q \\ 2 \nmid \alpha, \alpha>1}} p^{\alpha},
$$

$k \geq 2$ an odd integer, $\chi$ a primitive character modulo $q$, and $m$ any positive integer such that $\chi^{m}$ and $\chi^{k m}$ are also primitive characters modulo $q$. If $(k m, q)=1$, then we have the identity

$$
\begin{aligned}
\sum_{a_{1}=1}^{q} \cdots \sum_{a_{k-1}=1}^{q} \chi\left(a_{1}^{m} \cdots a_{k-1}^{m}\left(1-a_{1}-\cdots-a_{k-1}\right)^{m}\right) \\
= \begin{cases}q^{(k-1) / 2} \bar{\chi}\left(k^{k m}\right)\left(\frac{k}{q}\right) \prod_{p \mid q} \varepsilon_{p}^{k-1} & \text { if } 2 \nmid q, \\
q^{(k-1) / 2} \bar{\chi}\left(k^{k m}\right)\left(\frac{2}{k}\right)\left(\frac{k}{q}\right) \prod_{\substack{p \mid q \\
p \neq 2}} \varepsilon_{p}^{k-1} & \text { if } 2 \mid q .\end{cases}
\end{aligned}
$$

Corollary 2. Let $q \geq 3$ be a perfect square, $k \geq 2$ an integer, $\chi$ any primitive character modulo $q$, and $m$ any positive integer such that $\chi^{m}$ and $\chi^{k m}$ are also primitive characters modulo $q$. If $(k m, q)=1$, then we have the identity

$$
\sum_{a_{1}=1}^{q} \cdots \sum_{a_{k-1}=1}^{q} \chi\left(a_{1}^{m} \cdots a_{k-1}^{m}\left(1-a_{1}-\cdots-a_{k-1}\right)^{m}\right)=q^{(k-1) / 2} \bar{\chi}\left(k^{k m}\right) .
$$

From our theorems, we can see that the estimate in Katz [3] is the best possible. For general modulus $q$, whether there exists a similar formula is an open problem.

2. Some lemmas. To prove the theorems, we need some lemmas. For convenience, we define the Gauss sums $G(m, \chi)$ as follows:

$$
G(m, \chi)=\sum_{a=1}^{q} \chi(a) e(a m / q)
$$


where $e(y)=e^{2 \pi i y}$. For $m=1$, we write $\tau(\chi)=\sum_{a=1}^{q} \chi(a) e(a / q)$. The various properties and applications of $\tau(\chi)$ can be found in many analytic number theory books (see [1] and [4]). Here we first use the properties of Gauss sums to prove the following:

Lemma 1. Let $p$ be a prime, $\alpha$ a positive integer, and $\chi$ a primitive character modulo $p^{\alpha}$. Then for any positive integers $n_{1}, \ldots, n_{k}(k \geq 2)$ such that $\chi^{n_{1}}, \ldots, \chi^{n_{k}}$ and $\chi^{n_{1}+\cdots+n_{k}}$ are all primitive characters modulo $p^{\alpha}$, we have the identity

$$
\begin{aligned}
& \tau\left(\chi^{n_{1}}\right) \cdots \tau\left(\chi^{n_{k}}\right) \\
& \quad=\tau\left(\chi^{n_{1}+\cdots+n_{k}}\right) \sum_{a_{1}=1}^{p^{\alpha}} \cdots \sum_{a_{k-1}=1}^{p^{\alpha}} \chi\left(a_{1}^{n_{1}} \cdots a_{k-1}^{n_{k-1}}\left(1-a_{1}-\cdots-a_{k-1}\right)^{n_{k}}\right) .
\end{aligned}
$$

Proof. Using the properties of the trigonometric sums and Gauss sums, we can write

$$
\begin{aligned}
\sum_{a_{1}=1}^{p^{\alpha}} \cdots & \sum_{a_{k-1}=1}^{p^{\alpha}} \chi\left(a_{1}^{n_{1}} \cdots a_{k-1}^{n_{k-1}}\left(1-a_{1}-\cdots-a_{k-1}\right)^{n_{k}}\right) \\
= & \sum_{\substack{a_{1}=1 \\
a_{1}+\cdots+a_{k} \equiv 1\left(\bmod p^{\alpha}\right)}}^{p^{\alpha}} \cdots \sum_{a_{k}=1}^{p^{\alpha}} \chi\left(a_{1}^{n_{1}} \cdots a_{k}^{n_{k}}\right) \\
= & \frac{1}{p^{\alpha}} \sum_{a_{1}=1}^{p^{\alpha}} \cdots \sum_{a_{k}=1}^{p^{\alpha}} \chi\left(a_{1}^{n_{1}} \cdots a_{k}^{n_{k}}\right) \sum_{u=1}^{p^{\alpha}} e\left(\frac{u\left(a_{1}+\cdots+a_{k}-1\right)}{p^{\alpha}}\right) \\
= & \frac{1}{p^{\alpha}} \tau\left(\chi^{n_{1}}\right) \cdots \tau\left(\chi^{n_{k}}\right) \overline{\tau\left(\chi^{n_{1}+\cdots+n_{k}}\right)} .
\end{aligned}
$$

Then Lemma 1 follows from the identity $\left|\tau\left(\chi^{n_{1}+\cdots+n_{k}}\right)\right|^{2}=p^{\alpha}$.

Lemma 2. Let $q=q_{1} q_{2}$ with $\left(q_{1}, q_{2}\right)=1$. Then for any character $\chi$ modulo $q$, there exists a unique character $\chi_{i}$ modulo $q_{i}(i=1,2)$ such that $\chi=\chi_{1} \chi_{2}$ and

$$
\begin{aligned}
\sum_{a_{1}=1}^{q} \cdots & \sum_{a_{k-1}=1}^{q} \chi\left(a_{1}^{n_{1}} \cdots a_{k-1}^{n_{k-1}}\left(1-a_{1}-\cdots-a_{k-1}\right)^{n_{k}}\right) \\
= & \left(\sum_{a_{1}=1}^{q_{1}} \cdots \sum_{a_{k-1}=1}^{q_{1}} \chi_{1}\left(a_{1}^{n_{1}} \cdots a_{k-1}^{n_{k-1}}\left(1-a_{1}-\cdots-a_{k-1}\right)^{n_{k}}\right)\right) \\
& \times\left(\sum_{b_{1}=1}^{q_{2}} \cdots \sum_{b_{k-1}=1}^{q_{2}} \chi_{2}\left(b_{1}^{n_{1}} \cdots b_{k-1}^{n_{k-1}}\left(1-b_{1}-\cdots-b_{k-1}\right)^{n_{k}}\right)\right) .
\end{aligned}
$$


Proof. From the properties of the reduced residue system modulo $q$ we have

$$
\begin{aligned}
\sum_{a_{1}=1}^{q} \cdots \sum_{a_{k-1}=1}^{q} \chi & \left(a_{1}^{n_{1}} \cdots a_{k-1}^{n_{k-1}}\left(1-a_{1}-\cdots-a_{k-1}\right)^{n_{k}}\right) \\
= & \sum_{a_{1}=1}^{q_{1}} \cdots \sum_{a_{k-1}=1}^{q_{1}} \sum_{b_{1}=1}^{q_{2}} \cdots \sum_{b_{k-1}=1}^{q_{2}} \chi_{1} \chi_{2} \\
& \times\left(\left(a_{1} q_{2}+b_{1} q_{1}\right)^{n_{1}} \cdots\left(a_{k-1} q_{2}+b_{k-1} q_{1}\right)^{n_{k-1}}\right. \\
& \left.\times\left[1-\left(a_{1} q_{2}+b_{1} q_{1}\right)-\cdots-\left(a_{k-1} q_{2}+b_{k-1} q_{1}\right)\right]^{n_{k}}\right) \\
= & \left(\sum_{a_{1}=1}^{q_{1}} \cdots \sum_{a_{k-1}=1}^{q_{1}} \chi_{1}\left(a_{1}^{n_{1}} \cdots a_{k-1}^{n_{k-1}}\left(1-a_{1}-\cdots-a_{k-1}\right)^{n_{k}}\right)\right) \\
& \times\left(\sum_{b_{1}=1}^{q_{2}} \cdots \sum_{b_{k-1}=1}^{q_{2}} \chi_{2}\left(b_{1}^{n_{1}} \cdots b_{k-1}^{n_{k-1}}\left(1-b_{1}-\cdots-b_{k-1}\right)^{n_{k}}\right)\right) .
\end{aligned}
$$

This proves Lemma 2 .

Lemma 3. Let $p$ be a prime, $\alpha$ a positive integer, $q=p^{\alpha}$, and $\chi a$ primitive character modulo $q$. Then for any positive integer $m$ with $(m, p)$ $=1$, we have the identity

$$
\tau^{m}(\chi)= \begin{cases}q^{(m-1) / 2} \tau\left(\chi^{m}\right) \bar{\chi}\left(m^{m}\right) & \text { if } 2 \mid \alpha, \\ q^{(m-1) / 2} \varepsilon_{p}^{m-1}\left(\frac{m}{p}\right) \tau\left(\chi^{m}\right) \bar{\chi}\left(m^{m}\right) & \text { if } 2 \nmid m \alpha \text { and } p>2, \\ q^{(m-1) / 2}\left(\frac{2}{m}\right) \tau\left(\chi^{m}\right) \bar{\chi}\left(m^{m}\right) & \text { if } p=2 \text { and } 2 \nmid \alpha .\end{cases}
$$

Proof. We first introduce the hyper-Kloosterman sum by

$$
K(q, m+1, z)=\sum_{\substack{x_{1}, \ldots, x_{m} \bmod q \\\left(x_{1}, p\right)=\cdots=\left(x_{m}, p\right)=1}} e\left(\frac{x_{1}+\cdots+x_{m}+z \bar{x}_{1} \cdots \bar{x}_{m}}{q}\right)
$$

for $q=p^{\alpha}, m \geq 1$, and $p \nmid z$. Define an exponential sum by

$$
I(q, m, z)=\sum_{\substack{x \bmod q \\(x, p)=1}} e\left(\frac{m x+z \bar{x}^{m}}{q}\right) .
$$

From R. A. Smith [5] or Yangbo Ye [6] we know that 
(3)

$$
\begin{aligned}
& K(q, m+1, z) \\
& \quad= \begin{cases}q^{(m-1) / 2} I(q, m, z) & \text { if } 2 \mid \alpha \text { and } p>2, \\
q^{(m-1) / 2} \varepsilon_{p}^{m-1}\left(\frac{2^{m-1} z^{m-1} m}{p}\right) I(q, m, z) & \text { if } 2 \nmid m \alpha \text { and } p>2, \\
q^{(m-1) / 2}\left(\frac{2}{m}\right)^{\alpha} I(q, m, z) & \text { if } p=2 .\end{cases}
\end{aligned}
$$

Now for any primitive character $\chi$ modulo $q=p^{2 \alpha}$, note that

$$
\left(\frac{(2 z)^{m-1}}{p}\right)=\left(\frac{2 z}{p}\right)^{m-1}=1
$$

if $2 \nmid m$ and $p>2$ with $(p, z)=1$. From (3), the definition and properties of Gauss sums we have

$$
\begin{aligned}
& \sum_{z=1}^{q} \chi(z) K(q, m+1, z) \\
& \quad= \begin{cases}q^{(m-1) / 2} \sum_{z=1}^{q} \chi(z) I(q, m, z) & \text { if } 2 \mid \alpha \text { and } p>2, \\
q^{(m-1) / 2} \varepsilon_{p}^{m-1}\left(\frac{m}{p}\right) \sum_{z=1}^{q} \chi(z) I(q, m, z) & \text { if } 2 \nmid m \alpha \text { and } p>2, \\
q^{(m-1) / 2}\left(\frac{2}{m}\right)^{\alpha} \sum_{z=1}^{q} \chi(z) I(q, m, z) & \text { if } p=2 .\end{cases}
\end{aligned}
$$

Note that $\tau(\chi) \neq 0$ and

$$
\sum_{z=1}^{q} \chi(z) K(q, m+1, z)=\tau^{m+1}(\chi)
$$

and

$$
\sum_{z=1}^{q} \chi(z) I(q, m, z)=\tau(\chi) \tau\left(\chi^{m}\right) \bar{\chi}\left(m^{m}\right) .
$$

Combining this with (4) we immediately get Lemma 3.

3. Proof of the theorems. From the three lemmas above, we can easily prove the theorems. First we prove Theorem 1 . Let $q=p^{\alpha}$ with $2 \nmid \alpha$, and let $\chi$ be a primitive character modulo $q$. If $p>2$, then for any positive odd number $k \geq 2$ and positive integers $n_{1}, \ldots, n_{k}$ with $\left(n_{1} \cdots n_{k}\left(n_{1}+\cdots\right.\right.$ $\left.\left.+n_{k}\right), q\right)=1$, from Lemma 3 we have

$$
\begin{aligned}
\tau^{n_{1}}(\chi) \cdots \tau^{n_{k}}(\chi)= & q^{\frac{n_{1}+\cdots+n_{k}-k}{2}} \varepsilon_{p}^{n_{1}+\cdots+n_{k}-k}\left(\frac{n_{1} \cdots n_{k}}{p}\right) \\
& \times \tau\left(\chi^{n_{1}}\right) \cdots \tau\left(\chi^{n_{k}}\right) \bar{\chi}\left(n_{1}^{n_{1}} \cdots n_{k}^{n_{k}}\right)
\end{aligned}
$$


and

$$
\begin{aligned}
\tau^{n_{1}}(\chi) \cdots \tau^{n_{k}}(\chi)= & \tau^{n_{1}+\cdots+n_{k}}(\chi) \\
= & q^{\frac{n_{1}+\cdots+n_{k}-1}{2}} \varepsilon_{p}^{n_{1}+\cdots+n_{k}-1}\left(\frac{n_{1}+\cdots+n_{k}}{p}\right) \\
& \quad \times \tau\left(\chi^{n_{1}+\cdots+n_{k}}\right) \bar{\chi}\left(\left(n_{1}+\cdots+n_{k}\right)^{n_{1}+\cdots+n_{k}}\right) .
\end{aligned}
$$

Therefore, by Lemma 1 we get

$$
\begin{aligned}
q^{\frac{n_{1}+\cdots+n_{k}-1}{2}} \varepsilon_{p}^{n_{1}+\cdots+n_{k}-1}( & \left.\frac{n_{1}+\cdots+n_{k}}{p}\right) \\
& \times \tau\left(\chi^{n_{1}+\cdots+n_{k}}\right) \bar{\chi}\left(\left(n_{1}+\cdots+n_{k}\right)^{n_{1}+\cdots+n_{k}}\right) \\
= & q^{\frac{n_{1}+\cdots+n_{k}-k}{2}} \varepsilon_{p}^{n_{1}+\cdots+n_{k}-k}\left(\frac{n_{1} \cdots n_{k}}{p}\right) \\
& \times \tau\left(\chi^{n_{1}}\right) \cdots \tau\left(\chi^{n_{k}}\right) \bar{\chi}\left(n_{1}^{n_{1}} \cdots n_{k}^{n_{k}}\right) \\
= & q^{\frac{n_{1}+\cdots+n_{k}-k}{2}} \varepsilon_{p}^{n_{1}+\cdots+n_{k}-k}\left(\frac{n_{1} \cdots n_{k}}{p}\right) \tau\left(\chi^{n_{1}+\cdots+n_{k}}\right) \bar{\chi}\left(n_{1}^{n_{1}} \cdots n_{k}^{n_{k}}\right) \\
& \times \sum_{a_{1}=1}^{p^{\alpha}} \sum_{p_{k-1}^{\alpha}=1}^{n^{\alpha}} \chi\left(a_{1}^{n_{1}} \cdots a_{k-1}^{n_{k-1}}\left(1-a_{1}-\cdots-a_{k-1}\right)^{n_{k}}\right) .
\end{aligned}
$$

Noting that $\tau\left(\chi^{n_{1}+\cdots+n_{k}}\right) \neq 0$, from (5) we obtain

$$
\begin{aligned}
\sum_{a_{1}=1}^{p^{\alpha}} \cdots \sum_{a_{k-1}=1}^{p^{\alpha}} \chi\left(a_{1}^{n_{1}} \cdots\right. & \left.a_{k-1}^{n_{k-1}}\left(1-a_{1}-\cdots-a_{k-1}\right)^{n_{k}}\right) \\
= & q^{(k-1) / 2} \varepsilon_{p}^{k-1}\left(\frac{\left(n_{1}+\cdots+n_{k}\right) n_{1} \cdots n_{k}}{p}\right) \\
& \times \bar{\chi}\left(\left(n_{1}+\cdots+n_{k}\right)^{n_{1}+\cdots+n_{k}}\right) \chi\left(n_{1}^{n_{1}} \cdots n_{k}^{n_{k}}\right) .
\end{aligned}
$$

For the case of $p=2$, by the same argument we can also get

$$
\begin{aligned}
\sum_{a_{1}=1}^{p^{\alpha}} \cdots \sum_{a_{k-1}=1}^{p^{\alpha}} \chi\left(a_{1}^{n_{1}} \cdots\right. & \left.a_{k-1}^{n_{k-1}}\left(1-a_{1}-\cdots-a_{k-1}\right)^{n_{k}}\right) \\
= & q^{(k-1) / 2}\left(\frac{2}{\left(u_{1}+\cdots+u_{k}\right) n_{1} \cdots n_{k}}\right) \\
& \times \bar{\chi}\left(\left(n_{1}+\cdots+n_{k}\right)^{n_{1}+\cdots+n_{k}}\right) \chi\left(n_{1}^{n_{1}} \cdots n_{k}^{n_{k}}\right) .
\end{aligned}
$$

This proves Theorem 1 for the case $q=p^{\alpha}$; then from Lemma 2 we easily get the general conclusion. This proves Theorem 1.

Using the same methods we can also prove Theorem 2. This completes the proof of the theorems. 
Acknowledgments. The authors express their gratitude to the referee for his/her very helpful and detailed comments.

\section{References}

[1] T. M. Apostol, Introduction to Analytic Number Theory, Springer, New York, 1976.

[2] D. A. Burgess, On Dirichlet characters of polynomials, Proc. London Math. Soc. 13 (1963), 537-548.

[3] N. M. Katz, Estimates for "nonsingular" multiplicative character sums, Inter. Math. Res. Not. 7 (2002), 333-349.

[4] Chengdong Pan and Chengbiao Pan, Elements of the Analytic Number Theory, Science Press, Beijing, 1991.

[5] R. A. Smith, On n-dimensional Kloosterman sums, J. Number Theory 11 (1979), 324-343.

[6] Y. B. Ye, Estimation of exponential sums of polynomials of higher degrees II, Acta Arith. 93 (2000), 221-235.

[7] W. P. Zhang and W. L. Yao, A note on the Dirichlet characters of polynomials, ibid. 115 (2004), 225-229.

[8] W. P. Zhang and Y. Yi, On Dirichlet characters of polynomials, Bull. London Math. Soc. 34 (2002), 469-473.

Department of Mathematics

Northwest University

Xi'an, Shaanxi, P.R. China

E-mail: wpzhang@nwu.edu.cn

zfxu@nwu.edu.cn

Received on 20.5.2004

and in revised form on 26.8.2005 